\title{
Spatial Orientations of Angular Momentum Vectors of Galaxies in Supercluster $S$ [247+040+0029] and Substructures
}

\author{
J. R. Malla, W. Saurer, B. Aryal
}

Journal of Nepal Physical Society

Volume 7, Issue 1, April 2021

(Special Issue: ANPA Conference, 2020)

ISSN: 2392-473X (Print), 2738-9537(Online)

\section{Editors:}

Dr. Santosh KC

San Jose State University, USA (Editor in Chief) Dr. Pashupati Dhakal

Thomas Jefferson National Accelerator Facility, USA Dr. Yadav Pandit

Baptist Health Science University, USA

Managing Editor:

Dr. Binod Adhikari

St. Xavier's College, Kathmandu, Nepal

JNPS, 7 (1), 39-48 (2021)

DOI: http://doi.org/10.3126/jnphyssoc.v7i1.36974

Published by:

Nepal Physical Society

P.O. Box: 2934

Tri-Chandra Campus

Kathmandu, Nepal

Email: npseditor@gmail.com

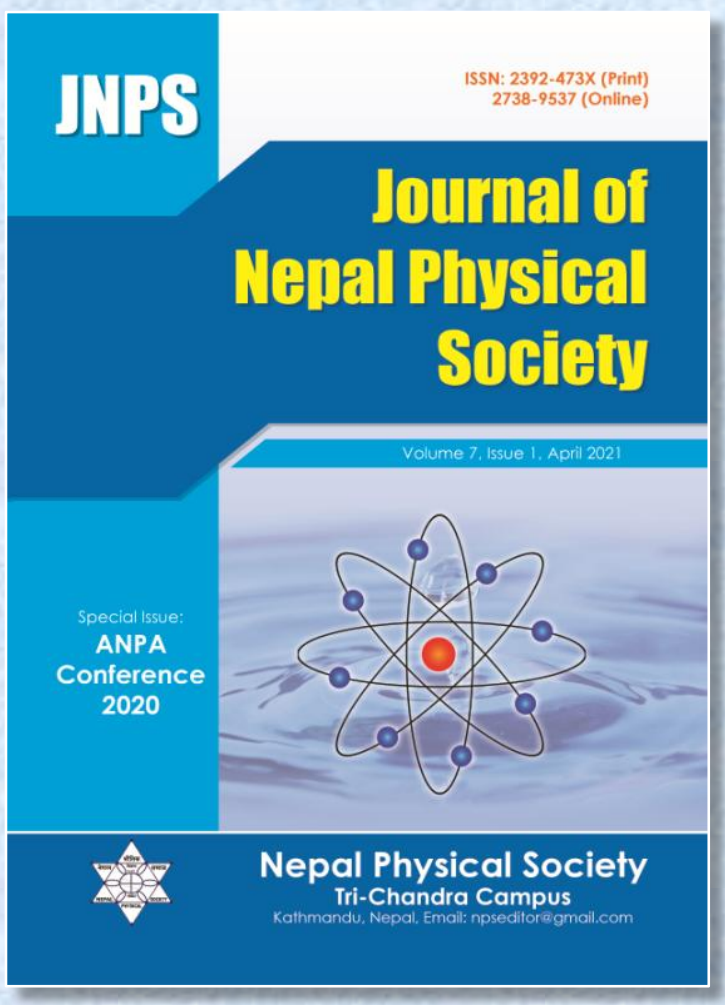




\title{
Spatial Orientations of Angular Momentum Vectors of Galaxies in Supercluster S [247+040+0029] and Substructures
}

\author{
J. R. Malla ${ }^{1, *}$, W. Saurer ${ }^{2}$, B. Aryal ${ }^{1}$ \\ ${ }^{1}$ Central department of Physics, Kirtipur, Kathmandu, Nepal \\ ${ }^{2}$ Institute of Astro-particle Physics, Innsbruck University, Austria \\ *Corresponding Email: janak.745711@cdp.tu.edu.np
}

Received: 10 August, 2020; Revised: 15 February, 2021; Accepted: 19 February, 2021

\begin{abstract}
We present an analysis of spatial orientations of 1331 galaxies in the supercluster $S$ [247+040+0029]. The main goal of this work is to search for a new substructure candidate and examine the orientation of angular momentum in the framework of three different scenarios (hierarchy, pancake, and primordial vorticity models) using "position angle-inclination method", and to test the dependence on magnitude with the angular momentum orientations and their projections. We identified five substructures studying number density contour map with considerably high concentration of galaxies. Using random simulation method to remove the selection effect on database, we carried out expected isotropic distribution using cosmological principle. We then used three statistical tests: Chi-square, Auto-correlation, and Fourier to identify isotropy between observed and expected isotropic distributions. We varied radius in the range $0.3^{\circ}$ to $1.0^{\circ}$ to find substructures in supercluster. From this we found five sub-structures when taking radius $0.3^{\circ}$ and two substructures for $1.0^{\circ}$ radius in the number density counter maps of galaxies. By analyzing the result obtained through statistical tests, we are able to conform that evolution of galaxy in the supercluster and substructures supports "Hierarchy model" giving the angular momentum of galaxies in the large scale structure tends to be oriented random with respect to the reference coordinate system.
\end{abstract}

Keywords: Galaxies: evolution, Supercluser, Substructure, Orientation.

\section{INTRODUCTION}

The galaxy clusters are gravitationally bound largescale structures of multiple galaxies. The evolution of these galaxy clusters is determined by time and scenario of formation of nature of change of their structures and constituents. Weizacker [1951] and Gamow [1939] postulated the rotation of galaxies might be a clue of physical conditions under which these systems formed. Thus, understanding the distribution of spatial orientation of the spin vectors of galaxies is crucial to understanding the origin of the angular momenta of galaxies.

There are number of contradictory theoretical models predicting the various galaxy cluster formation scenarios. The 'hierarchy model' (Peebles, 1969) predicts that the direction of angular momentum is entirely random. According to this scenario, galaxies were first formed and then obtained their angular momentum by tidal forces while they were gathering gravitationally to form a cluster with no dissipation. The pancake model'(Doroshkevich, 1973; Doroshkevich \& Shandarin, 1978) predicts that the angular momentum of galaxies tend to lie within the cluster plane whereas the 'primordial vorticity model'(Ozernoy, 1978; Stein 1974) says that the angular momentum of galaxies tend to be oriented perpendicular to the cluster plane. Lee and Pen (2002) found the observational evidence for the alignment of galaxy spin axes with the local tidal share field. Their result is consistent with the linear tidal-torque model based on gravitational instability. In an analytical study, in order to concluded a large-scale coherence in the orientation of galaxies in the two-dimension sheet like structures in the frame of the tidal-torque theory. 
According to some scenarios of cosmological structure evolution, the orientation of galactic axes in clusters should favor a certain direction while in other scenarios galaxies are expected to be randomly oriented. Attempts to reveal any deviation from isotropy have been performed over the past years with different and sometimes contradictory results. The work done before 1985 is described by MacGillivray and Dodd (1985), they pointed out that, most studies agree on a random galactic distribution within the local super cluster (LSC) plane, however they suggested that the galactic planes can be oriented preferentially parallel to the LSC plane at some distance from it. In a paper Kapranidis and Sullivan (1983) analyzed samples of bright spirals belonging to the LSC and found no strong evidence for alignment of these galaxies.

However, Jaaniste and Saar (1977) claimed the existence of mean perpendicularity of galactic planes with respect to the LSC plane. Since the earlier approaches were based mostly on analysis of highly inclined and edge-on galaxies, Jaaniste and Saar took all galaxies into consideration, including the face on ones. This approach was critically discussed and modified by Flin and Godlowski (1986). These authors and later Godlowski (1993 1994) analyzed large samples within the LSC and come to the conclusion that there exists a preferential orientation of galactic planes perpendicular to the LSC plane and that there is evidence for aligning the galactic rotation axes along the direction toward the Virgo cluster centre.

In the present study, we are interested to find our angular momentum vectors of all galaxies in a supercluster S [247+040+0029] and look at the number density map to find the possibilities of substructures there. Finally, we intend to correlate the orientation of galaxies in those substructures with the whole supercluster.

\section{DATABASE}

In this work, we have chosen a supercluster S[247+040+0029] whose all photometric data came from SDSS survey (York et. al., 2000). We received the SDSS database through our collaboration with Prof. Walter Saurer's group at the institute of Astroparticle physics, Innsbruck University, Austria. This supercluster includes 1331 galaxies out of which we excluded number of galaxies in our database based. Holmberg (1946) suggested a value of $\mathrm{q}^{*}=0.2$ for oblate spheroid and $\mathrm{q}^{*}=0.13$ for elliptals. At first, we used $\mathrm{q}^{*}=0.2$ to find inclination angle, we used $\mathrm{q}^{*}=0.13$ for those galaxies which did not give inclination angle for $\mathrm{q}^{*}=0.2$. Finally we have excluded those galaxies (5 galaxies) which do not give inclination angle for $\mathrm{q}^{*}=0.13$. Thus, we have 1326 galaxies in our sample of which position (Right Ascension and Declination), ratio of minor and major diameter, position angles in $\mathrm{r}$-filter of all SDSS passbands are available.

\section{GODLOWSKIAN TRANSFORMATION}

The Polar angle $(\rho)$ between the galactic spin vectors and a reference plane, and the Azimuthal angle $(\phi)$ between the projection of a galactic spin vectors onto the reference plane and $\mathrm{x}$-axis within this plane are used to view the three dimensional (spatial) orientation of the spin vector $\mathrm{SV}$ of a galaxy (Flin, \& Godlowski, 1986). These angles are given by the equations

$$
\begin{gathered}
\sin \theta=-\operatorname{cosisin} \alpha \pm \sin i \sin p \cos \delta \\
\sin \varphi=(\cos \theta)^{-1}[-\cos i \cos \delta \sin \alpha+\sin i(\mp \sin p \sin \delta \sin \alpha \mp \cos p \cos \alpha)]
\end{gathered}
$$

Here $\mathrm{i}, \boldsymbol{\alpha}, \boldsymbol{\delta}$ and $\mathrm{P}$ represent the inclination angle, declination, right ascension and position angles respectively.

The inclination angle (i) is the angle between the normal to the galaxy plane and the observers lineof-sight and can be calculated by using the formula Holmberg (1946),

$$
\cos ^{2} i=(b / a)^{2}-\frac{q *^{2}}{1-q *^{2}} \quad 3
$$

Here $q^{*}$ represents the intrinsic flatness factor of the galaxy. The intrinsic flatness of a disk galaxy depends on the morphological type and is taken from Heidmann et. al. (1972). Any selection effect on the parameters (e.g. positions, position angles, inclination angle etc) in the right hand side of equation $(1,2)$ plays a major role in determining the expected isotropic distributions of polar angle $(\nabla)$ and azimuthal angle $(\phi)$. This effect can be observed in the inclination angle distribution also. 
Thus in our database different kinds of selection effects can be noticed. To remove such selection effects, we use the method proposed by Aryal and Saurer (2000).

\section{METHOD OF ANALYSIS}

To reduce selection effects, we use the method proposed by Aryal \& Saurer (2000) the spatial distribution of the galaxy rotation axis is assumed to be isotropic. Theoretically, the isotropic distribution of polar angle is cosine and that of azimuthal angle is the average distribution curve with the restriction that the database is free from selection effect. We run simulations in order to define expected isotropic distribution curves for both the theta and phi distributions. The isotropic distribution curves are based on simulations generating $10^{7}$ virtual galaxies.

Here we describe the procedure for the removal the selection effects to obtain the isotropic
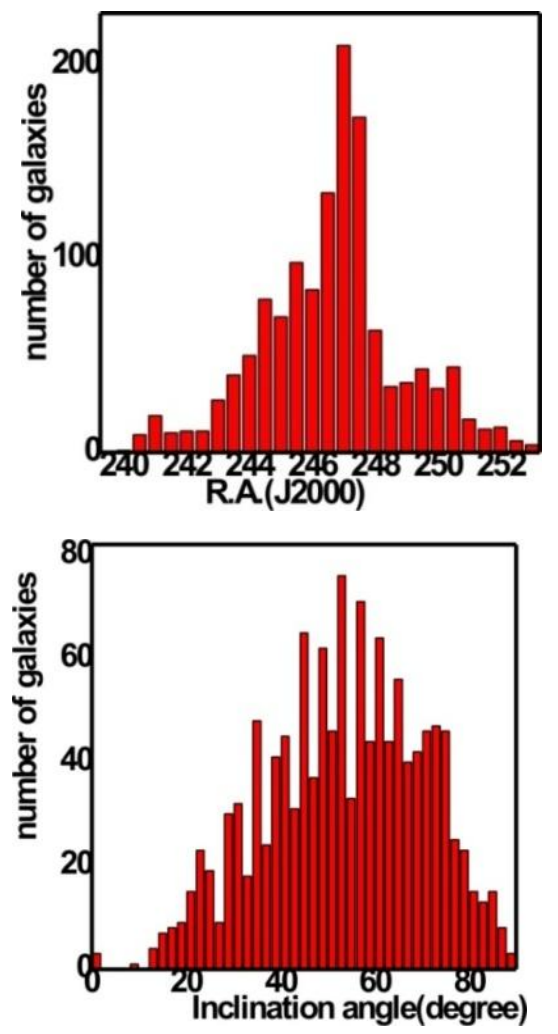

distributions for both $\nabla$ and $\phi$ as given by the Aryal \& Saurer (2000). We present the process of numerical simulation for the galaxies in the sample S [247+040+0029]. At first we observed the distributions of $\alpha, \delta, \mathrm{P}$ and $\mathrm{i}$ for the galaxies in the sample S[247+040+0029]. Fig. 1 shows the distributions of $\alpha, \delta, \mathrm{P}$ and $\mathrm{i}$ for the galaxies in the sample S [247+040+0029]. The histograms represent the number of observed galaxies. We see that in the fig. 1 the right ascension $(\alpha)$ is randomly distributed from $240.09^{\circ} \equiv$ to $252.97^{\circ}$ in 24 bins of bin size $0.56^{\circ}$. The declination $\delta$ is randomly distributed in the range $32.85 \equiv$ to $45.15^{\circ}$ in 42 bins of bin size $0.3^{\circ}$ (Fig. 1). The distribution of the position angle $\mathrm{P}$ (Fig. 1) is little homogeneous and distributed in the range $90^{\circ}$ to $240^{\circ}$ in 34 bins of bin size $10^{\circ}$. And there is again an inhomogeneous distribution of the inclination angle $\mathrm{i}$ in the range $1^{\circ}$ to $89^{\circ}$ in 23 bins of bin size $4^{\circ}$ (Fig. 1).
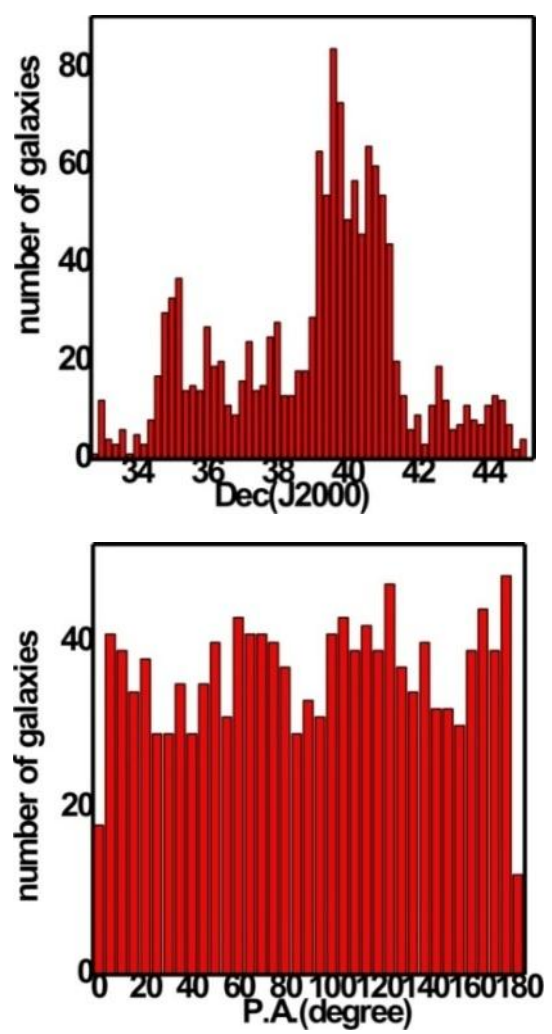

Figure 1: The distributions of right ascension $(\alpha)$, declination $(\delta)$, position angle (P) and inclination angle (i) of the galaxies in the sample S [247+040+0029]. The y-axes of histograms represent the number of observed galaxies.

\section{RESULTS \& DISCUSSION}

The all sky distribution of galaxies of our database in supercluster $\mathrm{S}$ [247+040+0029] is shown in Fig 2, which indicates the inhomogeneous distribution of galaxies. We can see that, almost all the galaxies were found to be grouped in the different region of space of supercluster. 


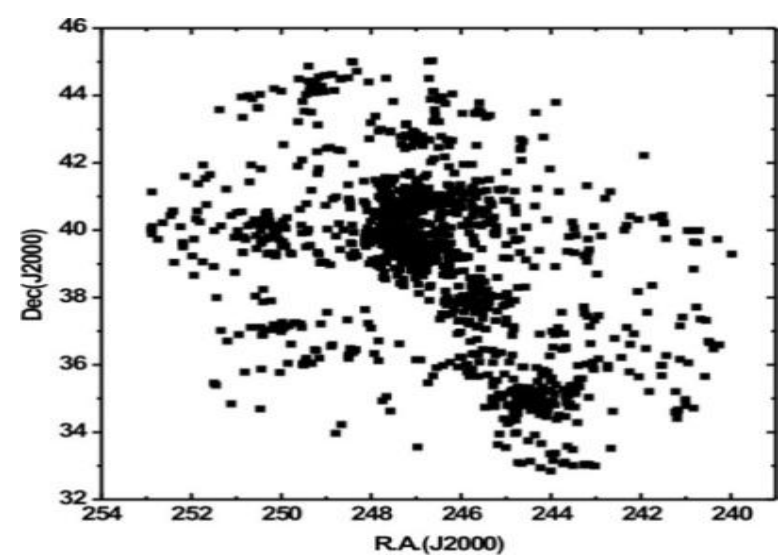

Fig. 2: All sky distribution of total galaxies of superclusters $\mathrm{S}[247+040+0029]$ in the equatorial co-ordinate system. The apparent distribution of the galaxies is because of the nature of the survey $\left(7^{\text {th }}\right.$ data release).
At first, we discuss the distribution of polar $(\nabla)$ and azimuthal ( $\phi)$ angles of galaxy rotation axes in $r-$ filter in de Vaucouleurs system of supercluster $\mathrm{S}$ [247+040+0029]. Any deviation from expected isotropic distribution will be tested using four statistical parameters, namely chi-square probability $\left(\mathrm{P}>\chi^{2}\right)$, auto-correlation coefficient $(\mathrm{C} / \mathrm{C}$ $(\sigma)$ ), first order Fourier coefficient $\left(\Delta_{11} / \sigma\left(\Delta_{11}\right)\right)$ and first order Fourier probability $\left(\mathrm{P}>\Delta_{1}\right)$. For anisotropy, the limit of chi-square probability $\mathrm{P}$ $\left(>\chi^{2}\right)$ is $<0.050$, auto-correlation coefficient $(\mathrm{C} / \mathrm{C}$ $(\sigma))$ is $>1.0$, first order Fourier coefficient $\left(\Delta_{11} / \sigma\right.$ $\left.\left(\Delta_{11}\right)\right)$ is $>1.5$ and Fourier probability $\mathrm{P}\left(>\Delta_{1}\right)$ is $<0.150$ respectively. These statistical limits were proposed by the Godlowski (1993-1994) in galaxy orientation studies.

Table 1: Statistical parameters in the polar and azimuthal angles of galaxies in the Supercluster S[247 $+040+0029]$ and its sub- structures (first column). The chi-square probability $P>\chi^{2}$ ) is given in the second column. The third and fourth columns show first order Fourier coefficient $\left(\Delta_{11} / \sigma\left(\Delta_{11}\right)\right)$ and Fourier probability $P\left(>\Delta_{1}\right)$ the last column tests the auto co-relation coefficient $(C / C(\sigma))$.

\begin{tabular}{|l|l|l|l|l|}
\hline \multicolumn{1}{|c|}{ Sample } & \multicolumn{1}{|c|}{$\left(\mathbf{P}\left(>\chi^{2}\right)\right.$} & \multicolumn{1}{|c|}{$\left(\boldsymbol{\Delta}_{\mathbf{1 1}} / \boldsymbol{\sigma}\left(\boldsymbol{\Delta}_{\mathbf{1 1}}\right)\right)$} & \multicolumn{1}{|c|}{$\mathbf{P}\left(>\boldsymbol{\Delta}_{\mathbf{1}}\right)$} & \multicolumn{1}{|c|}{$(\mathbf{C} / \mathbf{C}(\boldsymbol{\sigma}))}$. \\
\hline Polar Angle & & & & \\
\hline S [247+040+0029] & 0.511 & -0.180 & 0.954 & -0.747 \\
\hline S1[247+040+0029] & 0.943 & -0.316 & 0.952 & -1.186 \\
\hline S2[247+040+0029] & 0.909 & -0.123 & 0.987 & -0.886 \\
\hline S3[247+040+0029] & 0.893 & 0.179 & 0.982 & -0.584 \\
\hline S4[247+041+0029] & 0.996 & -0.327 & 0.871 & -0.139 \\
\hline S5[244+035+0029] & 0.822 & -0.003 & 0.983 & -1.098 \\
\hline Azimuthal angle & & & & \\
\hline S [1247+040+0029] & 0.954 & 0.401 & 0.633 & -2.206 \\
\hline S1[247+040+0029] & 0.570 & -0.260 & 0.855 & -1.041 \\
\hline S2[247+040+0029] & 0.911 & 0.166 & 0.964 & -0.059 \\
\hline S3[247+040+0029] & 0.969 & -0.102 & 0.966 & -0.028 \\
\hline S4[247+041+0029] & 0.838 & -0.469 & 0.804 & -0.906 \\
\hline S5[244+035+0029] & 0.081 & 1.383 & 0.303 & -0.405 \\
\hline
\end{tabular}

The supercluster S [247+040+0029] from SDSS catalogue consists of 1331 galaxies out of which five galaxies have been omitted because of unclear position angles. As shown in table1, The values of chi-square probability $\left(\mathrm{P}\left(>\chi^{2}\right)\right.$, first order Fourier coefficient $\left(\Delta_{11} / \sigma\left(\Delta_{11}\right)\right)$, first order Fourier probability $\mathrm{P}\left(>\Delta_{1}\right)$ and auto-correlation coefficient $(\mathrm{C} / \mathrm{C}(\sigma))$ obtained for the polar angle $(\nabla)$ are $0.511,-0.180,0.954 \&-0.747$ respectively. Here, all statistics shows the isotropic distribution of spin vectors in the supercluster.

We also study the 'humps' (bins with more solutions than the expected) and 'dips' (bins with less solutions than the expected) in the polar and azimuthal angle distributions in addition to the statistical tests. In the plot of the $\nabla$-distribution (see Fig.3a as an example), solid curve represents the expected isotropic distribution where as dashed 
curve is the cosine distribution. The solid circles with $\pm 1 \quad \sigma$ error bars represent the observed distribution. A dip (or hump) at $\nabla<45^{\circ}$ suggests that the spin vectors of galaxies tend to orient perpendicular (or parallel) with respect to the equatorial coordinate system. Similarly, a hump (or dip) in the larger $\nabla\left(\nabla>45^{\circ}\right)$ indicates that the spin vectors of galaxies tend to be oriented perpendicular with respect to the equatorial coordinate system. In Fig.3a there is a hump on polar angle distribution at $\nabla=55^{\circ}$. This hump is due to 13 more observed solutions than expected solutions in the range $\nabla>45^{\circ}$.

For the plot of azimuthal $\phi$-distribution, the statistics value obtained for the chi-square probability $\left(\mathrm{P}>\chi^{2}\right)$, first order Fourier coefficient $\left(\Delta_{11} / \sigma\left(\Delta_{11}\right)\right)$, first order Fourier probability ( $\mathrm{P}>$ $\left.\Delta_{1}\right)$ and auto-correlation coefficient $(\mathrm{C} / \mathrm{C}(\sigma))$ are $0.950,0.401,0.633$ and -2.206 respectively. Here, all statistical tests show strong isotropic distribution of spin vectors.

In the figure $3 b$, solid curve represents the expected isotropic distribution whereas dashed

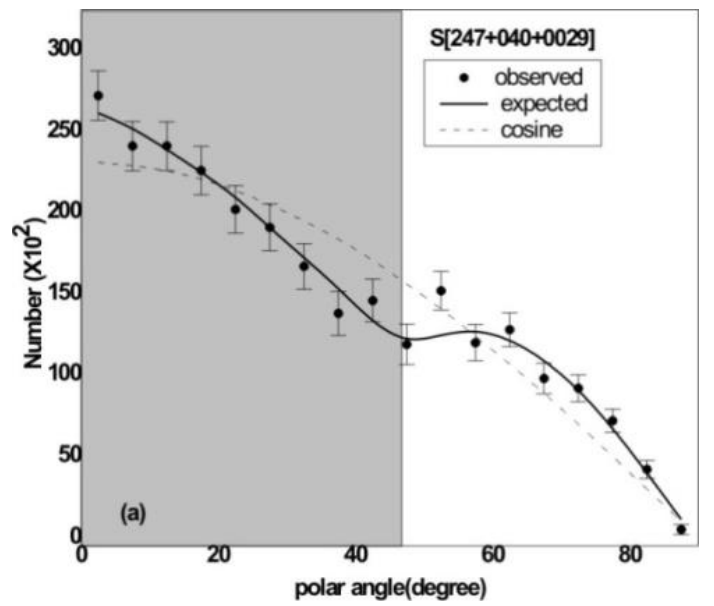

curve is the average distribution. The solid circles with $\pm 1 \quad \sigma$ error bars represent the observed distribution. The humps and dips in the plots of $\phi$-distribution are not so easy to interpret as compared to $\nabla$-distribution. It is because the range of $\phi$ is $-90^{\circ}$ to $+90^{\circ}$. In the plot of the $\phi$-distribution, $\phi=0^{\circ}$ means spin vector projections tend to point radially towards the center of the equatorial coordinate system. A hump in the middle (central eight bins) of the plot suggests that the spin vector projections of galaxies tend to point towards the center of the chosen co-ordinate system. Similarly, a hump at first four and last four bins indicates that the spin vectors projections of galaxies tend to be oriented tangentially with respect to the chosen reference co-ordinate system.

There are two small dip in the plot of azimuthal angle at $\phi=-75^{\circ}$ and at $\phi=55^{\circ}$, but they are insignificant and they are due to difference in number of observed solutions than that of expected. So, we can conclude that there is no preferred alignment of spin-vectors of galaxies.

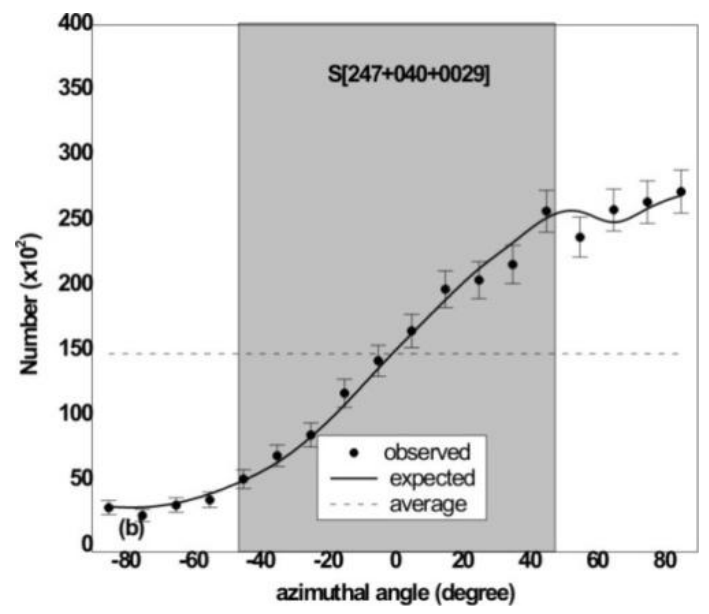

Fig. 3: (a) Polar and (b) azimuthal angle distribution of galaxies in supercluster S [247+040+0029]. The dashed line represents theoretical cosine distribution for polar angle and average distribution for azimuthal angle, B-spline represents expected isotropic distribution obtained through simulation and solid circles with $\pm 1 \sigma$ error bars represents observed distribution of polar and azimuthal angle.

The substructure S1 [247+040+0029] that we have chosen from the contour map of number density of galaxies of total supercluster (SDSS catalogue consists of 1365 galaxies) consist of 500 galaxies. By statistics, the values of chi-square probability $\left(\mathrm{P}>\chi^{2}\right)$, first order Fourier coefficient $\left(\Delta_{11} / \sigma\left(\Delta_{11}\right)\right)$, first order Fourier probability $\left(\mathrm{P}>\Delta_{1}\right)$ and autocorrelation coefficient $(\mathrm{C} / \mathrm{C}(\sigma))$ obtained for the polar angle $(\rho)$ are found to be $0.943,-0.316,0.952$
\&-1.186, respectively. Here, all statistics shows the distribution of spin vectors is strong isotropic distribution. For the plot of azimuthal $\phi$ distribution, the statistics value obtained for chisquare probability $\left(\mathrm{P}>\chi^{2}\right)$, first order Fourier coefficient $\left(\Delta_{11} / \sigma\left(\Delta_{11}\right)\right)$, first order Fourier probability $\left(\mathrm{P}>\Delta_{1}\right)$ and auto-correlation coefficient $(\mathrm{C} / \mathrm{C}(\sigma))$ are $0.570,-0.260,0.855$ and -1.041 , respectively. 

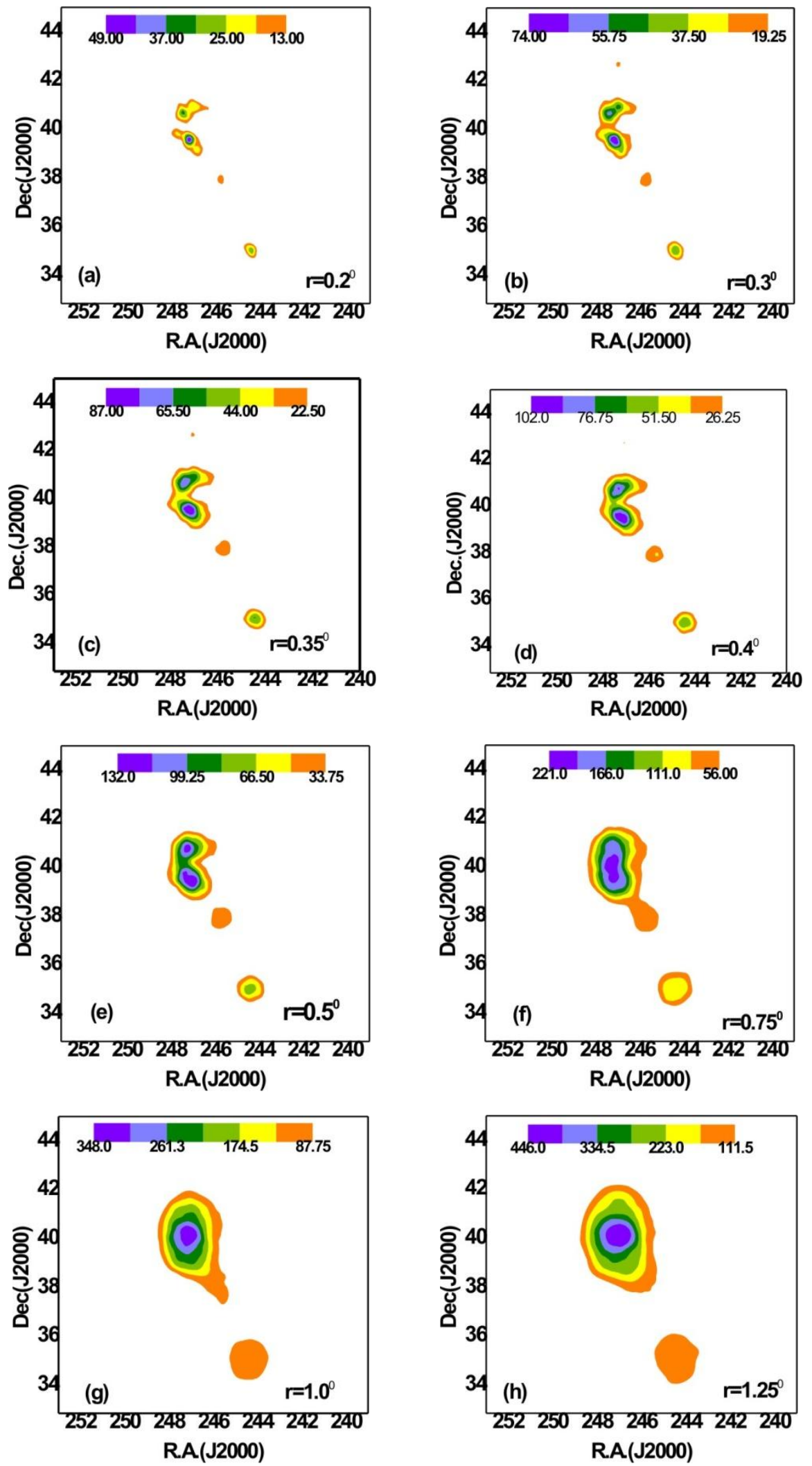

Figure 4: Number density map of galaxies in the Supercluster $\mathrm{S}[247+040+0029]$ having nearest neighbor distance of each galaxies at (a) $r=0.2^{\circ}$, (b) $r=0.3^{\circ}$, (c) $r=0.35^{\circ}$, (d) $r=0.4^{\circ}$ (a) $r=0.5^{\circ}$, (b) $r=0.75^{\circ}$, (c) $r=1.0^{\circ}$, (d) $r=1.25^{\circ}$ and The color bar is shown. As the radius increases, the subclustering becomes prominent. 
In the Fig 5a, the number of observed solution that have $\mathrm{P}<45^{\circ}$ is found to be 699 and that for expected solutions are 670 . The difference between observed and expected solution in that range is 1 . At this region, there is one dip at $22^{\circ}$. For the large angles $\left(\mathrm{P}>45^{\circ}\right)$, the number of expected solutions are less by 1 than that of observed. Again there is one dip at $47^{\circ}$ in that range. These dips are due to local effect. Thus, we conclude no preferred alignment of spin vectors of galaxies.

In the azimuthal angle distribution as shown in Fig $5 b$, the observed solutions for range $-45^{\circ}<\phi<+45^{\circ}$ are found to be 556, whereas the expected solutions are 549. This shows that observed solutions are more than the expected solution by 7 . There is a significant dip at $38^{\circ}$. For $\phi<-45^{\circ}$, observed solution are 49 and that for expected are 52 that is observed solutions are 3 less than expected. Due to difference in observed and expected solutions, there is a dip at $-55^{\circ}$ in this range. For the large angles $(\phi$ $>45^{\circ}$ ), the observed 395 solutions are 4 less than what was expected. There are no hump and dip in this range. So we can conclude that there is no preferred alignment among spin-vectors of galaxies.

The substructure $S 2[247+040+0029]$ that we have chosen from the contour map of number density of galaxies of total supercluster (SDSS catalogue consists of 1331 galaxies) consist of 188 galaxies. From statistics, the values of chi-square probability $\left(\mathrm{P}>\chi^{2}\right)$, first order Fourier coefficient $\left(\Delta_{11} / \sigma\right.$ $\left.\left(\Delta_{11}\right)\right)$, first order Fourier probability $\left(\mathrm{P}>\Delta_{1}\right)$ and auto-correlation coefficient $(\mathrm{C} / \mathrm{C}(\sigma))$ obtained for the polar angle $(\rho)$ are found to be $0.909,-0.123$, 0.984\&-0.886 respectively. Here, all statistics shows the strong isotropic distribution of spin vectors of galaxies. For the plot of azimuthal $\phi$ distribution, the statistics value obtained for the chisquare probability $\left(\mathrm{P}>\chi^{2}\right)$, first order Fourier coefficient $\left(\Delta_{11} / \sigma\left(\Delta_{11}\right)\right)$, first order Fourier probability $\left(\mathrm{P}>\Delta_{1}\right)$ and auto-correlation coefficient $(\mathrm{C} / \mathrm{C}(\sigma))$ are $0.911, \quad 0.166,0.964 \&-0.059$ respectively. In the Fig 5c, the numbers of observed solution are equal to that of expected solutions. At this region, there is one dip at $18^{\circ}$. For the large angles $\left(\mathrm{P}>45^{\circ}\right)$, there is again one significant dip at $67^{\circ}$. These dips are may due to local effects. Thus, we conclude that there is no preferred alignment of spin vectors of galaxies.

In the azimuthal angle distribution as shown in Fig $5 \mathrm{~d}$, there is no significant hump and dip. We again conclude that there is no preferred alignment among spin-vectors of galaxies.
The substructure S3[244+0035+0029] that we have chosen from the contour map of number density of galaxies of total supercluster (SDSS catalogue consists of 1365 galaxies) consist of 149 galaxies. By statistics, the values of chi-square probability $\left(\mathrm{P}>\chi^{2}\right)$, first order Fourier coefficient $\left(\Delta_{11} / \sigma\left(\Delta_{11}\right)\right)$, first order Fourier probability $\left(\mathrm{P}>\Delta_{1}\right)$ and autocorrelation coefficient $(\mathrm{C} / \mathrm{C}(\sigma))$ obtained for the polar angle $(\rho)$ are found to be $0.893,0.179,0.982$ $\&-0.584$ respectively. Here, all statistics shows the distribution of spin vectors in large supercluster is strong isotropic. For the plot of azimuthal $\phi$ distribution, the statistics value obtained for the chisquare probability $\left(\mathrm{P}>\chi^{2}\right)$, first order Fourier coefficient $\left(\Delta_{11} / \sigma\left(\Delta_{11}\right)\right)$, first order Fourier probability $\left(\mathrm{P}>\Delta_{1}\right)$ and auto-correlation coefficient $(\mathrm{C} / \mathrm{C}(\sigma))$ are $0.969,-0.102, \quad 0.966 \&-0.028$ respectively. In the Fig 5e, the number of observed solution that have $\mathrm{P}<45^{\circ}$ is found to be 206 and that for expected solutions are 208 .

The difference between observed and expected solution in that range is 2 . At this region, there is one dip at $13^{\circ}$. For the large angles $\left(\mathrm{P}>45^{\circ}\right)$, the number of expected solutions are less by 2 than that of observed and there is one significant hump at $48^{\circ}$ in that range which cancel the dip observed at lower angles. Thus, we conclude no preferred alignment of spin vectors of galaxies.

In the azimuthal angle distribution as shown in Fig 5f, the observed solutions for range $-45^{\circ}<\phi<+45^{\circ}$ are found to be 161 , whereas the expected solutions are 165. This shows that observed solutions are less than the expected solution by 4 . For $\phi<-45^{\circ}$, observed solution are 12 and that for expected are 11 that is observed solutions are more than expected by 1 . For the large angles $\left(\phi>45^{\circ}\right)$, the observed 125 solutions are 3 more than what was expected. There is one significant dip at $-75^{\circ}$ which cancels the significant hump at $-65^{\circ}$. But there is no more hump and dip in other range so we can conclude that there is no preferred alignment among spin-vectors of galaxies.

The substructure S4 [247+0041+0029] that we have chosen from the contour map of number density of galaxies of total supercluster (SDSS catalogue consists of 1331 galaxies) consist of 134 galaxies. By statistics, the values of chi-square probability $\left(\mathrm{P}>\chi^{2}\right)$, first order Fourier coefficient $\left(\Delta_{11} / \sigma\left(\Delta_{11}\right)\right)$, first order Fourier probability $\left(\mathrm{P}>\Delta_{1}\right)$ and autocorrelation coefficient $(\mathrm{C} / \mathrm{C}(\sigma))$ obtained for the polar angle $(\rho)$ are found to be $0.996,-0.327$, $0.871 \&-0.139$ respectively. Here, all statistics shows the isotropic distribution of spin vectors. For 

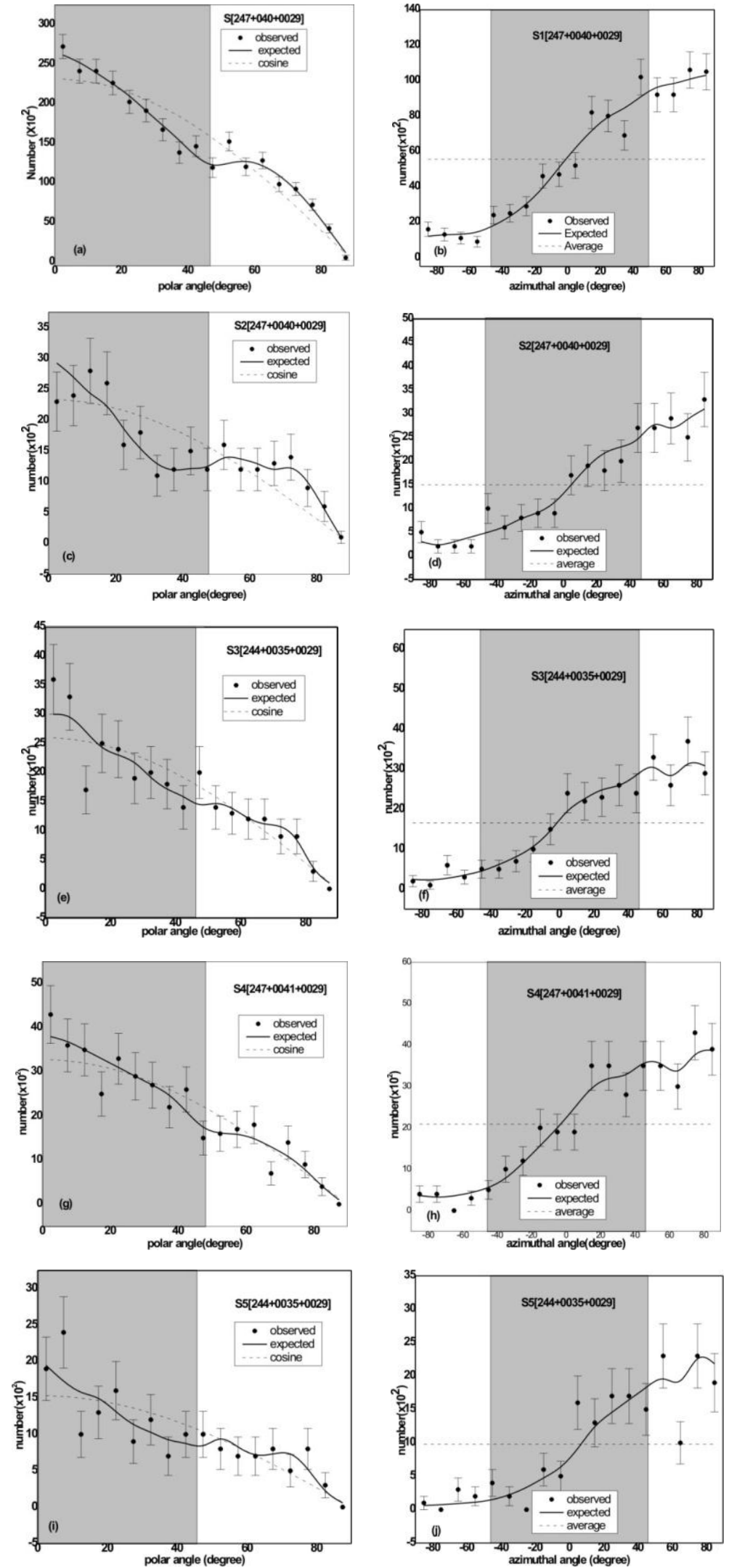

Fig. 5: $(a, c, e, g, i)$ The polar $(P)$ and $(b, d, f, h, j)$ azimuthal angle $(\phi)$ distributions of galaxies in the substructure of supercluster $S[247+040+0029]$. The solid line represents the expected isotropic distributions. The cosine and average distributions (dashed) are shown for the comparison. The solid circles with $\pm 1 \sigma$ error bars represent the observed distribution. 
the plot of azimuthal $\phi$-distribution, the statistics value obtained for the chi-square probability (P> $\left.\chi^{2}\right)$, first order Fourier coefficient $\left(\Delta_{11} / \sigma\left(\Delta_{11}\right)\right)$, first order Fourier probability $\left(\mathrm{P}>\Delta_{1}\right)$ and autocorrelation coefficient $(\mathrm{C} / \mathrm{C}(\sigma))$ are 0.838 , $0.469,0.804 \&-0.906$ respectively. In the Fig $5 \mathrm{~g}$, the number of observed solution that have $\mathrm{P}<45^{\circ}$ is found to be 173 and that for expected solutions are 174. There is no significant difference between observed and expected solution in that range. At this region, there is one dip at $3^{\circ}$. For the large angles $\mathrm{P}>45^{\circ}$, the number of expected solutions are less by 1 than that of observed. So there is no preferred alignment of spin vectors of galaxies.

In the azimuthal angle distribution as shown in Fig $5 \mathrm{~h}$, the observed solutions for range $-45^{\circ}<\phi<+45^{\circ}$ are found to be 143 , whereas the expected solutions are 142. This shows that observed solutions exceeded the expected solution by 1 . For $\phi<-45^{\circ}$, observed and expected solutions both are 11. For the large angles $\left(\phi>45^{\circ}\right)$, the observed 114 solutions are 1 less than what was expected. There is a significant hump at $-45^{\circ}$. It is due to local effect but there are no other significant humps and dips. We again conclude that there is no preferred alignment among spin-vectors of galaxies.

The substructure S5[244+0035+0029] that we have chosen from the contour map of number density of galaxies of total supercluster (SDSS catalogue consists of 1365 galaxies) consist of 88 galaxies. By statistics, the values of chi-square probability $\left(\mathrm{P}>\chi^{2}\right)$, first order Fourier coefficient $\left(\Delta_{11} / \sigma\left(\Delta_{11}\right)\right)$, first order Fourier probability $\left(\mathrm{P}>\Delta_{1}\right)$ and autocorrelation coefficient $(\mathrm{C} / \mathrm{C}(\sigma))$ obtained for the polar angle $(\theta)$ are found to be 0.822 , $0.003,0.983 \&-1.098$ respectively. Here, all statistics shows the distribution of spin vectors in large supercluster is strong isotropic distribution. For the plot of azimuthal $\phi$-distribution, the statistics value obtained for the chi-square probability $\left(\mathrm{P}>\chi^{2}\right)$, first order Fourier coefficient $\left(\Delta_{11} / \sigma\left(\Delta_{11}\right)\right)$, first order Fourier probability $\left(\mathrm{P}>\Delta_{1}\right)$ and auto-correlation coefficient $(\mathrm{C} / \mathrm{C}(\sigma))$ are $0.081,1.383,0.303$ \&0.405 respectively.

In the Fig $5 \mathrm{i}$, the number of observed solution that have $\theta<45^{\circ}$ is found to be 120 and that for expected solutions are also 120 . At this region, there is a hump at $9^{\circ}$ and a dip at $13^{\circ}$ due to local effects. For the large angles $\left(\nabla>45^{\circ}\right)$, there is no significant hump and dip in that range. Thus, we conclude no preferred alignment of spin vectors of galaxies.

In the azimuthal angle distribution as shown in Fig $5 \mathrm{j}$, the observed solutions for range $-45^{\circ}<\phi<+45^{\circ}$ are found to be 95 , whereas the expected solutions are 88. This shows that observed solutions are more than the expected solution by 7 . For $\phi<-45^{\circ}$, observed solution are 6 and that for expected are 3 that is observed solutions are 3 more than expected. For the large angles $\left(\phi>45^{\circ}\right)$, the observed 75 solutions are 9 less than what was expected. There is one significant hump at $5^{\circ}$ and a dip at $65^{\circ}$. It is due to local effect. We again conclude that there is no preferred alignment among spin-vectors of galaxies.

\section{CONCLUSION}

We have studied the spatial orientation of spin vectors of SDSS galaxies in supercluster $S$ [247+040+0029] containing 1331 galaxies. The method involved for this study is proposed by Flin and Godlowski (1986). To remove selection effect and obtain theoretically expected isotropic distribution, we randomly generated $10^{7}$ virtual galaxies through numerical simulation using the method suggested by Aryal \& Saurer (2000). Furthermore, using the galaxies density map to identify substructure, three eminent substructures, namely S1 [247+040+0029], S2[247+040+0029], $\mathrm{S} 3[244+0035+0029]$, for radius value 0.3 degree and two substructures, namely S4 $[247+0041+0029]$ and S5[244 +0035+0029] for radius value 1degree has been found. Three statistical tools are used to check whether the distribution of galactic plane throughout the supercluster and in identified sub-structure is isotropic or not.

The main conclusions of our work are numbered as below:

- In this work, no preferred alignment of spin vectors of galaxies is obtained in the supercluster $\mathrm{S}[247+040+0029]$, so there is no anisotropic distribution. This suggests that galactic rotation axes favor the "hierarchy model" as suggested by Peebles (1969) of galactic evolution within the supercluster. Same trend are found in the substructure evolution. However, local effect can be seen in some sub-structure suggesting gravitational shearing effect.

- We also found no preferred alignment of angular momentum vectors of galaxies in any of the substructures S1 [247+040+0029], S2 $[247+040+0029], \quad S 3 \quad[244+0035+0029], \quad$ S4 $[247+0041+0029]$ and S5[244 +0035+0029]. This suggests hierarchical evolution of galaxies in those substructures too. 
The lack of preferred alignment of galaxies in superclusters as well as in its substructures suggests the substructures are yet to evolve by merging and collisions through gravitation to obtain anisotropy in galactic orientation.

\section{ACKNOWLEDGEMENT}

One of the authors (J.R.Malla) wishes to express his sincere thanks to Astro-Particle Physics, Innsbruck University, Austria, for providing data access and the authorities of the SDSS for providing the database.

Editor's Note: This manuscript was submitted to Association of Nepali Physicists in America (ANPA) Conference 2020 for publication in special issue of Journal of Nepal Physical Society.

\section{REFERENCES}

[1] Aryal, B., \& Saurer, W. Comments on the expected isotropic distribution curves in the galaxy orientation studies. Astronomy and Astrophysics letter, 364: 97-100 (2000).

[2] Doroshkevich, A. G. The orientation of rotation of galaxies. Astrophysical Letters, 14: 11-13 (1973).

[3] Doroshkevich, A. G. \& Shandarin, S. F. Spatial structure of protoclusters and the formation of Galaxies. Monthly Notices Royal Astronomy Society, 184: 643-660 (1978).

[4] Flin, P. \& Godlowski, W. The Orientation of Galaxy Groups and formation of the local Supercluster. Monthly Notices Royal Astronomy Society, 222, 525 (1986).

[5] Gamow, G. \& Teller, E. On the origin of great Nebulae. Physics Review, 55: 654-657 (1939).

[6] Godlowski, W. Galactic orientation within the local Supercluster. Monthly Notices Royal Astron. Soc., 265: 874-880 (1993).

[7] Godlowski, W. Some aspects of the galactic orientation within the local Supercluster. Monthly Notices Royal Astronomy Society, 271: 19-30 (1994).

[8] Heidmann, J.; Heidmann, N. \& De vaucouleurs, G. Inclination and absorption effects on the apparent diameters,optical luminosities and neutral hydrogen radiation of galaxies-I.Optical and $21-\mathrm{cm}$ line data. Memories of the Royal Astronomical Society, 175: 85-104 (1972).

[9] Holmberg, E. On the apparent diameters and the orientation in space of extragalactic Nebulae. Meddelanden fran Lunds Astronomiska Observatorium Series II, 117: 3-82 (1946).

[10] Jaaniste, J. A.; Saar, E. M. An accretion theory of the spiral structure of galaxies. Pis'ma Astron.Zh. 3: 9-12 (1977).
[11] Kapranidis, S. \& Sulivan, W. T. The orientation in space of spiral galaxies in the local Supercluster. Astronomy and Astrophysics, 118: 33-38 (1983).

[12] Lee, J.; Pen, U. L. Detection of galaxy spin alignments in the point source catalog redshift survey shear field. The Astrophysical Journal Letters, 567(2): L111-L114 (2002).

[13] MacGillivray, H. T.; Dodd, R. J.The distribution of faint galaxies in a field of 15 square degrees near the south galactic pole. Monthly Notices of the Royal Astronomical Society, 193: 1-6 (1985).

[14] Ozernoy, L. M. The whirl theory of the origin of structure in the universe. IAU Symposium, 79: 427-438 (1978).

[15] Peebles, P. J. E. Origin of the angular momentum of Galaxies. The Astrophysical Journal, 155, 393 (1969).

[16] Stein, R. Galaxy formation from Primordial Turbulence. Astronomy \& Astrophysics, 35: 1729 (1974)

[17] Weizsaker, V. The evolution of Galaxies and stars. The Astrophysical Journal, 114: 165-186 (1951).

[18] York, D. G.; Adelman, J.; Anderson, J. E.; Anderson, S. F.; Annis, J.; Bahcall, N. A.; Bakken, J. A. \& 137 more. The sloan digital sky survey: Technical summary. The Astronomical Journal, 120: 1579-1587 (2000). 